Pacific Journal of Mathematics

CONTINUOUS DECOMPOSITIONS INTO CELLS OF 


\title{
CONTINUOUS DECOMPOSITIONS INTO CELLS OF DIFFERENT DIMENSIONS
}

\author{
JoHN J. WALSH AND DAVID C. WiLSON
}

In $1970 \mathrm{~S}$. Jones showed that the only continuous decomposition of $E^{n}$ into points and $k$-cells is the trivial decomposition into points. This is extended by showing that, for a pair of positive integers $r, s$ with $r+s \geq n$, the only continuous decomposition of $E^{n}$ into points, $r$-cells, and $s$-cells is the trivial decomposition into points.

1. Introduction. The structure of continuous decompositions of euclidean spaces is sufficiently rich to have supported investigations in both existence and non-existence directions. While the trivial decomposition into points, the decomposition consisting of the origin and boundary spheres of balls centered at the origin, and decompositions consisting of orbits of compact group actions provide examples of continuous decompositions of euclidean spaces, there are obstacles to easily describing examples displaying additional features. In 1952 R. D. Anderson exhibited a continuous decomposition of $E^{2}$ into nondegenerate cell-like subsets (i.e., nonseparating subcontinua of $E^{2}$ ) [Ann $]$ but showed that the decomposition elements could not be required to be, in addition, locally connected $\left[\mathbf{A n}_{\mathbf{2}}\right]$. (Surprisingly, such a decomposition can consist of mutually homeomorphic elements [L-W]). In 1955, E. Dyer established that the elements of such a decomposition cannot be decomposable [D. $\left.\mathbf{y}_{\mathbf{1}}\right]$ and shortly thereafter, extended the result to other dimensions by showing that there does not exist a nontrivial continuous decomposition of $E^{n}$ into points and 1-dimensional decomposable cell-like subsets $\left[\mathbf{D y}_{\mathbf{2}}\right]$. Similar analysis of decompositions with elements having dimension $>1$ awaited S. Jones' fundamental paper $\left[\mathbf{J o}_{\mathbf{2}}\right]$ that provided an adequate replacement for the role previously played by 0 -regularity, leading to the result that there does not exist a nontrivial continuous decomposition of $E^{n}$ into points and $k$-cells for any $k$. The last result is extended to

MAIN THEOREM. If $r+s \geq n$, then the only continuous decomposition of $E^{n}$ into points, $r$-cells, and s-cells is the trivial decomposition into points. 
A motivating factor for further pursuit of nonexistence results is that, in spite of extensive development of techniques for constructing continuous decompositions of $E^{n}\left(\left[\mathbf{W i}_{1}\right],\left[\mathbf{W i}_{2}\right],\left[\mathbf{W} \mathbf{a}_{1}\right],\left[\mathbf{W} \mathbf{a}_{2}\right]\right)$ and a strong approximation result in $\left[\mathbf{W a}_{3}\right]$, it remains a definite possibility that

Conjecture A. There does not exist a nontrivial continuous decomposition of $E^{n}$ into absolute retracts.

The conjecture is evidently true for $n=1$, is a consequence of previously mentioned results for $n=2$, and was recently established for $n=3$ in $[\mathbf{W}-\mathbf{W}]$. The techniques developed in [ $\left.\mathbf{W} \mathbf{a}_{3}\right]$ produce continuous decompositions of $E^{n}$ that preserve the global structure but not local structure of elements of the decomposition, in fact, they are so "1-dimensional" that Dyer's previously mentioned result $\left[\mathbf{D y}_{\mathbf{2}}\right]$ precludes the elements being decomposable. However, it is plausible that these techniques can be refined but the strategy that appears most promising, if successful, would do no better than

Conjecture B. For each integer $k$ with $2 k+5 \leq n$, there is a nontrivial continuous decomposition of $E^{n}$ consisting of cell-like sets that are homotopically (or, at least, homologically) locally connected through dimension $k$.

Appropriate formulations of these conjectures for upper semicontinuous decompositions of $E^{n}$ are settled by an example constructed by J. H. Roberts in 1936 [Ro] of an upper semicontinuous decomposition of $E^{2}$ each of whose elements is the homeomorphic copy of either a 1-cell or a letter " $H$ ". The decomposition can be adjusted and made to consist of 1-cells and 2-cells by carefully "blowing up" each letter " $H$ " to a 2-cell. While there is no upper semicontinuous decomposition of $E^{n}$ into 1-cells, $[\mathbf{R o} ; n=2]$ and $\left[\mathbf{J o}_{1} ; n \geq 3\right]$, a related unsettled problem is whether or not there is an upper semicontinuous decomposition of some euclidean space into $k$-cells for some $k \geq 2$.

2. Preliminaries. A decomposition $H$ of a space $X$ is upper semicontinuous provided the elements of $H$ are pairwise disjoint nonempty compact subsets and, for each open subset $U \subset X$, the set $\cup\{h \in H: h \subset U\}$ is open. The decomposition $H$ is continuous provided, in addition, for each open subset $U \subset X$, the set $U\{h \in H: h \cap U \neq \varnothing\}$ is open.

A map $f: X \rightarrow Y$ is proper provided $f^{-1}(C)$ is compact for each compact subset $C \subset Y$. Without further mention, we shall freely move 
between the equivalent settings of proper maps and upper semicontinuous decompositions and of proper open mappings and continuous decompositions.

The cohomological dimension with respect to a group $G$ of a space $X$ is denoted $D(X ; G)$; recall that $D(X ; G)$ equals the smallest integer $k \geq 0$ such that the inclusion induced homomorphism $i^{*}: \check{H}^{k}(X ; G) \rightarrow \check{H}^{k}(A ; G)$ is a surjection for each closed subset $A \subset X$. (Except in an appendix, the cohomology used is Čech theory.)

Two well documented facts are that $D(A ; G) \leq D(X ; G)$ for each $A \subset X$ and that $D(X ; \mathbf{Z})=\operatorname{dim} X$ whenever the covering $\operatorname{dimension} \operatorname{dim} X$ is finite.

A compact space $X$ is said to be $G$-acyclic provided it is connected and $\check{H}^{q}(X ; G)=0$ for $q \geq 1$. A proper map $f: X \rightarrow Y$ is $G$-acyclic provided each point inverse $f^{-1}(y)$ is $G$-acyclic.

3. Theorems of Jones and Dyer. The subsequent computations of cohomological dimension that comprise the proof of the Main Theorem are done by combining a result of $\mathrm{S}$. Jones that detects regular convergence in continuous collections of manifolds with a type of "Künneth formula" due to E. Dyer that detects cohomological dimension.

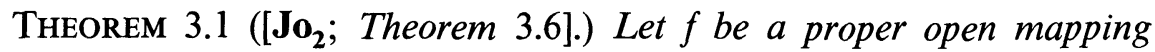
from a complete metric space $X$ onto a metric space $Y$ such that each $f^{-1}(y)$ is a compact orientable $k$-manifold. Then there is a dense $G_{\delta}$ subset $S \subset Y$ such that $f$ is $k$-regular at each interior point of $f^{-1}(s)$ for each $s \in S$.

The statement that follows is a variation of that in $\left[\mathbf{D y}_{3}\right]$ but the same proof applies.

THEOREM 3.2 ([Dy $\mathbf{y}_{3}$, Theorem 11]). Let $f$ be a mapping from a compact metric space $X$ onto a metric space $Y$ that is $k$-regular (over $\mathbf{Z}$ ) at a point $x \in f^{-1}(y)$ for some $y \in Y$; furthermore, assume that $D\left(f^{-1}(z) ; \mathbf{Z}\right) \leq k$ for each $z \in Y$, that $D(W ; \mathbf{Z})=m$ for each neighborhood $W$ of $y$, and that the rational cohomological dimension $D\left(f^{-1}(y) \cap U ; Q\right)=k$ for each closed neighborhood $U$ of $x$. Then $D(X, \mathbf{Z}) \geq m+k$.

4. Preliminary lemmas and computations. The lemmas are standard results; proofs are included for completeness.

LEMMA 4.1. If $f: E^{n} \rightarrow Y$ is a proper $G$-acyclic mapping, then $D(V ; G)$ $=n$ for each open subset $V \subset Y$. 
Proof. On the one hand, the Vietoris-Begle Mapping Theorem [Sp; p. 344] readily yields that $D(A ; G) \leq D\left(f^{-1}(A) ; G\right)$ and, hence, $D(A ; G) \leq n$ for each subset $A \subset Y$.

On the other hand, specifying a point $y \in V$, let $P \subset f^{-1}(V)$ be a compact polyhedral neighborhood of $f^{-1}(y)$; since $\check{H}^{n-1}(P ; G)$ is finitely generated and $f^{-1}(y)$ is $G$-acyclic, there is a neighborhood $V^{\prime}$ of $y$ with $f^{-1}\left(V^{\prime}\right) \subset P$ such that

$$
\operatorname{Im}\left\{i^{*}: \check{H}^{n-1}(P ; G) \rightarrow \check{H}^{n-1}\left(f^{-1}\left(V^{\prime}\right) ; G\right)\right\}=\{0\}
$$

and hence,

$$
\operatorname{Im}\left\{i^{*}: \check{H}^{n-1}\left(f^{-1}(V) ; G\right) \rightarrow \check{H}^{n-1}\left(f^{-1}\left(V^{\prime}\right) ; G\right)\right\}=\{0\} .
$$

For a compact neighborhood $N \subset V^{\prime}$ of $y, \check{H}^{n-1}\left(f^{-1}(\operatorname{Fr} N) ; G\right) \neq 0$ as the set $f^{-1}(\operatorname{Fr} N)$ separates $E^{n}$. We conclude that $i^{*}: \check{H}^{n-1}\left(f^{-1}(V) ; G\right) \rightarrow$ $\check{H}^{n-1}\left(f^{-1}(F r N) ; G\right)$ is not onto and, translating via the Vietoris-Begle Mapping Theorem, that $i^{*}: \check{H}^{n-1}(V ; G) \rightarrow \check{H}^{n-1}(\operatorname{FrN} ; G)$ is not onto and, consequently, $D(V ; G) \geq n$.

LEMMA 4.2. Let $f$ be a proper mapping from a metric space $X$ onto a metric space $Y$; for each positive integer $k$, the set $S(k)=\left\{y: \operatorname{dim} f^{-1}(y)\right.$ $\leq k\}$ is $a G_{\delta}$ subset of $Y$.

Proof. Let $n$ be a positive integer. For each $y \in S(k)$ specify a finite cover $\mathcal{Q}_{y}$ of $f^{-1}(y)$ such that each $U \in \mathcal{Q}_{y}$ is an open subset of $X$ with $\operatorname{diam} U<1 / n$ and every $k+2$ distinct elements of $\mathscr{Q}_{k}$ have empty intersection. Set $V_{n}=\left\{z: f^{-1}(z) \subset \cup \mathscr{Q}_{y}\right.$ for some $\left.y \in S(k)\right\}$; evidently, $S(k) \subset V_{n}$.

While the properness of $f$ assures that each $V_{n}$ is an open subset, the cover definition of dimension reveals that $\operatorname{dim} f^{-1}(z) \leq k$ for each $z \in$ $\cap V_{n}$; consequently, $S(k)=\cap V_{n}$.

Proposition 4.3. If $f$ is an open mapping from a compact metric space $X$ onto a metric space $Y$ and $f^{-1}(y)$ is a k-cell for each $y \in Y$, then $D(X ; \mathbf{Z}) \geq k+D(Y ; \mathbf{Z})$.

Proof. Set $m=D(Y ; \mathbf{Z})$ and assume that $Y$ has been replaced by a closed subset (if necessary) so that $D(W ; \mathbf{Z})=m$ for each nonempty open subset $W \subset Y$ (see Appendix I). An application of S. Jones' result recorded in Theorem 3.1 produces a point $y \in Y$ such that $f$ is $k$-regular at each interior point of $f^{-1}(y)$ and an application of E. Dyer's result recorded in Theorem 3.2 yields that $D(X ; \mathbf{Z}) \geq k+D(Y ; \mathbf{Z})$. 
COROLlARY 4.4. If $f: E^{n} \rightarrow Y$ is a proper open Z-acyclic mapping, $k$ is an integer $\geq 1$, and $K \subset Y$ is a compact subset with $f^{-1}(y)$ a $k$-cell for each $y \in K$, then $D(K ; \mathbf{Z})<n-k$.

Proof. The inequality $D(K ; \mathbf{Z}) \geq n-k$ together with Proposition 4.3 yield that $\operatorname{dim} f^{-1}(K)=D\left(f^{-1}(K) ; \mathbf{Z}\right) \geq n$ and, consequently, Int $f^{-1}(K) \neq \varnothing$. In turn, the openness of $f$ forces Int $K \neq \varnothing$ and, by Lemma $4.1, D(K ; \mathbf{Z})=n$. A second application of Proposition 4.3 produces the impossibility $D\left(f^{-1}(K) ; \mathbf{Z}\right) \geq k+n \geq n+1$.

5. Proof of main theorem. In establishing nonexistence results, it is preferable not to assume that the decomposition spaces are finite dimensional, a fact that is not known for upper semicontinuous or continuous decompositions of finite dimensional spaces into points and 1-cells. Since the computations that form the heart of the proof are cohomological, it is reasonable to expect that the Vietoris Begle Mapping Theorem assuring that the decomposition spaces have finite cohomological dimension is sufficient. While this is the case, one point in the proof requires a "general position" result recorded in Appendix II that can be avoided by assuming the decomposition space is finite dimensional.

Let $\pi: E^{n} \rightarrow E^{n} / H$ denote the quotient mapping induced by a continuous decompositin $H$ consisting of points, $r$-cells, and $s$-cells. For convenience, assume that $\pi^{-1} \pi(x) \neq$ point for each $x \in E^{n}$ (otherwise, apply the argument that follows to the restriction of $\pi$ to the open subset $\left\{x: \pi^{-1} \pi(x) \neq\right.$ point $\}$ of $\left.E^{n}\right)$.

Specify that $s<r, S=\left\{y \in E^{n} / H: \pi^{-1}(y)\right.$ is an $s$-cell $\}$, and that $R=\left\{y \in E^{n} / H: \pi^{-1}(y)\right.$ is an $r$-cell $\}$. Using Lemma $4.2, S$ is a $G_{\delta}$ subset of $E^{n} / H$ and, hence, $R$ is an $F_{\sigma}$ subset of $E^{n} / H$. Writing $R=\cup_{r=1}^{\infty} R_{i}$ where each $R_{i}$ is compact, Corollary 4.4 produces the inequality $D\left(R_{i} ; \mathbf{Z}\right)$ $\leq n-r-1$ and, since the Sum Theorem is valid for cohomological dimension, $D(R ; \mathbf{Z}) \leq n-r-1$. Recorded in a corollary in Appendix II is the "general position" result that there is a compact subset $K \subset$ $\left(E^{n} / H\right)-R$ with $D(K ; \mathbf{Z}) \geq n-D(R ; \mathbf{Z})-1 \geq r$. Since $K \subset S$, another application of Corollary 4.4 is that $D(K ; \mathbf{Z}) \leq n-s-1$. These last two inequalities combine to force $r+s \leq n-1$.

6. Decompositions into $(n-1)$-dimensional ANR's. Among the examples mentioned in the Introduction was the decomposition of $E^{n}$ consisting of the origin and boundary spheres of balls centered at the 
origin. The next result records that the non-vanishing of the $(n-1)$ dimensional cohomology of the boundary spheres is an attribute possessed by most elements of a continuous decomposition of an open subset of $E^{n}$ into $(n-1)$-dimensional absolute neighborhood retracts.

THEOREM 6.1. If $H$ is a continuous decomposition of an open subset of $E^{n}$ into absolute neighborhood retracts with $\operatorname{dim} h=n-1$ for each $h \in H$, then there is a dense $G_{\delta}$ subset $H^{\prime} \subset H$ such that $\check{H}^{n-1}\left(h^{\prime} ; \mathbf{Z}\right) \neq 0$ for each $h^{\prime} \in H^{\prime}$.

Proof. According to $\left[\mathrm{Jo}_{\mathbf{2}}\right.$; Corollary 2.5], there is a dense $G_{\delta}$ subset $H^{\prime} \subset H$ such that $H$ is 0 -regular at each element of $H^{\prime}$. We shall show that, for each component $C$ of each $h^{\prime} \in H^{\prime}$ with $\operatorname{dim} C=n-1$, $\check{H}^{n-1}(C ; \mathbf{Z}) \neq 0$.

Since $\operatorname{dim} C=n-1$, there is a point $x \in C$ and a connected open neighborhood $W$ of $x$ such that $N \backslash C$ is not connected for each neighborhood $N$ of $x$ contained in $W$. Since the decomposition $H$ is 0 -regular at $h^{\prime}$, there is an open neighborhood $V$ of $x$ contained in $W$ such that $h \cap V$ is contained in a component of $h \cap W$ whenever $h \in H$ and $h \cap V \neq \varnothing$.

Since the decomposition $H$ is continuous and is 0-regular at $h^{\prime}$, there is an open connected subset $U$ of the open set $U\{h \in H: h \cap V \neq \varnothing\}$ such that $h^{\prime} \cap U=C$ and $h \cap U$ is a component of $h$ whenever $h \cap U \neq$ $\varnothing$. The conclusion $\breve{H}^{n-1}(C ; \mathbf{Z}) \neq 0$ follows from Alexander Duality once we have shown that $U \backslash C$ (and, hence, $E^{n} \backslash C$ ) is not connected. Choose a separation $V \backslash C=V_{1} \cup V_{2}$ so that $V_{1}$ and $V_{2}$ are contained in different components of $W \backslash C$. Since no element of $H$ meets both $V_{1}$ and $V_{2}$, as $H$ is 0-regular at $h^{\prime}$, the open sets $U_{t}=\bigcup\left\{h \cap U: h \in H\right.$ and $\left.h \cap V_{i} \neq \varnothing\right\}$, $i=1,2$, form a separation of $U \backslash C$.

Appendix I. The next result is a consequence of the Sum Theorem that is valid for cohomological dimension; specifically, if $C$ is either a countable or a locally finite collection of closed subsets of $X$ and $D(C ; G)$ $\leq n$ for each $C \in \mathcal{C}$, then $D(\cup\{C \in \mathcal{C}) ; G \leq n$ (see [Ku]).

Proposition. If $X$ is a metric space and $D(X ; G) \geq n$, then $X$ contains a closed subset $A$ such that $D(U ; G) \geq n$ for each nonempty relatively open subset $U \subset A$.

Proof. Consider the collection $\mathfrak{V}$ of all open subsets of $V \subset X$ with $D(V ; G) \leq n-1$. Using paracompactness, there is a locally finite collec-

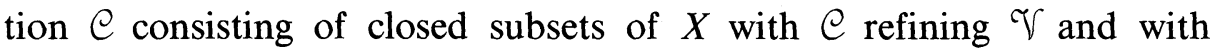


$\cup\{C \in \mathcal{C}\}=\cup\{V \in \mathbb{V}\}$. In particular, $D(C ; G) \leq n-1$ for each $C \in$ $\mathcal{C}$ and, therefore $D(\cup\{V \in \mathscr{V}\} ; G) \leq n-1$. Set $A=X-\cup\{V \in \mathscr{V}\}$. Since both $\cup\{V \in \mathcal{V}\}$ and each relatively open subset $U \subset A$ are $F_{\sigma}$ subsets of $X$, the inequality $D(U ; G) \leq n-1$ together with the Sum Theorem would lead to the impossibility $(\cup\{V \in \mathcal{V}\}) \cup U \in \mathcal{V}$.

Appendix II. Failing to know that the decomposition space associated with a cell-like decomposition of $E^{n}$ is finite dimensional necessitates the appendix. For, in the proof of the Main Theorem, if $E^{n} / H$ is finite dimensional, then the compact subset $K$ specified can be obtained by the following analysis.

Suppose that $X$ is a finite dimensional space and $R \subset X$ is a subset with $\operatorname{dim} R \leq k$. Using [Na; p. 32], specify a $G_{\delta}$ subset $R^{\prime} \subset X$ with $R \subset R^{\prime}$ and $\operatorname{dim} R^{\prime} \leq k$. The computation [HW; p. 28] states that $\operatorname{dim} X$ $\leq \operatorname{dim} R^{\prime}+\operatorname{dim}\left(X-R^{\prime}\right)+1$ and, hence, $\operatorname{dim}\left(X-R^{\prime}\right) \geq \operatorname{dim} X-k-$ 1. Since $X-R^{\prime}$ is an $F_{\sigma}$ subset of $X, X-R^{\prime}$ contains a closed (compact when $X$ is locally compact) subset $K$ of $X$ with $\operatorname{dim} K \geq \operatorname{dim} X-k-1$.

Apparently, neither of the results for covering dimension that guide the preceding argument are known for cohomological dimension. In the specialized setting of singular homology manifolds, duality arguments shall provide results that suffice for our purposes.

The Vietoris-Begle Mapping Theorem assures that the decomposition space $M^{n} / H$ associated with a cell-like decomposition $H$ of a $G$-orientable $n$-manifold is a $G$-orientable homology $n$-manifold ( $G$ is any abelian group). Since, in addition, $M^{n} / H$ is homotopically locally connected in every dimension (see [La]), each open subset is a homology $n$-manifold with respect to singular homology. In particular, for a relatively closed subset $A$ of an open subset $X \subset M^{n} / H$, there is the duality isomorphism

$$
H_{q}(X, X-A ; G) \cong \bar{H}_{c}^{n-q}(A ; G)
$$

where $\bar{H}_{c}^{*}$ is Alexander cohomology with compact supports (see [Sp., p. 342]). For computational purposes there is the equality

$$
\bar{H}_{c}^{q}(A ; G) \cong \check{H}^{q}\left(A^{+} ; G\right) \text { for } q \geq 1
$$

where $A$ is locally compact and $A^{+}$is the one point compactification (see [Sp., p. 322 and p. 334]). Furthermore, the homotopical local connectedness of $M^{n} / H$ permits the identification for a compact pair $(A, B)$

$$
\check{H}_{q}(A, B ; G)=\operatorname{Inv} \operatorname{Lim}\left\{H_{q}\left(U_{\alpha}, V_{\alpha} ; G\right)\right\}
$$


where $\left(U_{\alpha}, V_{\alpha}\right)$ ranges over pairs of open neighborhoods of $(A, B)$. The authors thank R. J. Daverman for his timely comments that aided the preparation of the appendix.

THEOREM. Let $H$ be an upper semicontinuous cell-like decomposition of a G-orientable n-manifold $M^{n}(\partial M=\varnothing)$ and let $R_{1}, R_{2}, \ldots$ be a sequence of closed subsets of $M^{n} / H$ with $D\left(R_{i} ; G\right) \leq k$ for each $i$. Then, for each $z \in H_{q}(U, V ; G)$ where $V \subset U$ are open subsets of $M^{n} / H$ and $q \leq n-k-$ 1 , there is a compact pair $(A, B)$ in $\left(M^{n} / H\right) \backslash \cup R_{i}$ such that $z \in \operatorname{Im}\left\{i_{*}\right.$ : $\left.\check{H}_{q}(A, B ; G) \rightarrow H_{q}(U, V ; G)\right\}$.

This supplies the essential ingredient for an analysis done subsequently that establishes the sought after.

COROllaRY. Let $H$ be an upper semicontinuous cell-like decomposition of an $n$-manifold $M^{n}(\partial M=\varnothing)$ and let $R_{1}, R_{2}, \ldots$ be a sequence of closed subsets of $M^{n} / H$ with $D\left(R_{i} ; \mathbf{Z}\right)<k$ for each $i$. Then there is a compact subset $K \subset\left(M^{n} / H\right) \backslash \cup R_{i}$ such that $D(K ; \mathbf{Z}) \geq n-k-1$.

Proof of Theorem. The first step is to establish that, for each $R_{i}$ and each pair of open subsets $(U, V)$, the inclusion induced homomorphisms $H_{q}\left(U \backslash R_{l}, V \backslash R_{i} ; G\right) \rightarrow H_{q}(U, V ; G)$ are isomorphisms for $q \leq n-k-2$ and surjective for $q=n-k-1$. For a locally compact subset $A \subset R_{i}$, the inequality $D(A ; G) \leq k$ and the Sum Theorem assure that $D\left(A^{+} ; G\right)$ $\leq k$. Therefore, $\check{H}^{q}\left(A^{+} ; G\right)=0$ for $q \geq k+1$ and, consequently, $\bar{H}^{q}(A ; G)=0$ for $q \geq k+1$. In turn, for $X$ equal to either $U$ or $V$, duality states that $H_{q}\left(X, X \backslash R_{i} ; G\right) \cong \bar{H}^{n-q}\left(X \cap R_{i} ; G\right)$ and, thus, $H_{q}\left(X, X \backslash R_{i} ; G\right)=0$ for $q \leq n-k-1$. Using the exact sequences for the pairs $\left(U, U-R_{i}\right)$ and $\left(V, V-R_{i}\right)$, we conclude that the inclusion induced homomorphisms $H_{q}\left(U \backslash R_{i} ; G\right) \rightarrow H_{q}(U ; G)$ and $H_{q}\left(V \backslash R_{i} ; G\right)$ $\rightarrow H_{q}(V ; G)$ are isomorphisms for $q \leq n-k-2$ and surjective for $q=n$ $-k-1$. Finally, the inclusion $\left(U \backslash R_{i}, V \backslash R_{i}\right) \hookrightarrow(U, V)$ induces homomorphisms between the exact sequences of the pairs and the "Five Lemma" yields that $H_{q}\left(U \backslash R_{i}, V \backslash R_{i} ; G\right) \rightarrow H_{q}(U, V ; G)$ is an isomorphism for $q \leq n-k-2$ and surjective for $q=n-k-1$.

Setting $\left(U_{0}, V_{0}\right)=(U, V), z_{0}=z$, and $R_{0}=\varnothing$, the proof is completed by recursively constructing a sequence of pairs of open sets $\left(U_{i}, V_{i}\right)$ and elements $z_{i} \in H_{q}\left(U_{i}, V_{i} ; G\right)$ satisfying:

(a) for $i \geq 1, \bar{U}_{i}$ is compact, $\bar{U}_{i} \subset U_{i-1}$, and $\bar{V}_{\imath} \subset V_{i-1}$; 
(b) $\bar{U}_{i} \cap\left(R_{0} \cup \cdots \cup R_{i}\right)=\varnothing$; and

(c) $z_{i-1}=i_{*}\left(z_{i}\right)$ where $i_{*}: H_{q}\left(U_{i}, V_{i} ; G\right) \rightarrow H_{q}\left(U_{i-1}, V_{i-1} ; G\right)$ is the inclusion induced homomorphism. Evidently, $(A, B)=\left(\cap \bar{U}_{l}, \cap \bar{V}_{i}\right)$ satisfies the conclusion of the Theorem.

Proof of Corollary. The inequality $D\left(R_{i} ; \mathbf{Z}\right) \leq k$ implies that $\check{H}^{q}(A, B ; \mathbf{Z})=0$ for each closed pair $(A, B)$ in $R_{i}$ and each integer $q \geq k+1$. An appeal to a Universal Coefficient Theorem [Sp, p. 336] reveals that $\check{H}^{q}(A, B ; \mathbf{Z} / 2 \mathbf{Z})=0$ for each closed pair $(A, B)$ in $R_{i}$ and each integer $q \geq k+1$ and, consequently, we conclude that $D\left(R_{i} ; \mathbf{Z} / 2 \mathbf{Z}\right)$ $\leq k$ for each $i$.

For each $q \leq n-1$, there is an open pair $(U, V)$ in $M^{n} / H$ with $H_{q}(U, V ; \mathbf{Z} / 2 \mathbf{Z}) \neq 0$. Otherwise, since $H_{q}(U, V ; \mathbf{Z} / 2 \mathbf{Z})$ and $H^{q}(U, V ; \mathbf{Z} / 2 \mathbf{Z})$ are isomorphic as $(\mathbf{Z} / 2 \mathbf{Z})$-vector spaces, we could conclude that $H^{q}(A, B ; \mathbf{Z} / 2 \mathbf{Z})=0$ for all closed pairs $(A, B)$ in $M^{n} / H$ and, consequently, $D\left(M^{n} / H ; \mathbf{Z} / 2 \mathbf{Z}\right) \leq q \leq n-1$. This contradicts that $D\left(M^{n} / H ; \mathbf{Z} / 2 \mathbf{Z}\right)=n($ see Lemma 4.1$)$.

Set $q=n-k-1$ and specify an open pair $(U, V)$ in $M^{n} / H$ with $H_{q}(U, V ; \mathbf{Z} / 2 \mathbf{Z}) \neq 0$. The Theorem produces a compact pair $(A, B)$ in $\left(M^{n} / H\right) \backslash \cup R_{i}$ with $\check{H}_{q}(A, B ; \mathbf{Z} / 2 \mathbf{Z}) \neq 0$. Taking limits with respect to the system of open pairs $\left(U_{\alpha}, V_{\alpha}\right)$ containing $(A, B)$, there is an embedding

$$
\begin{aligned}
\check{H}_{q}(A, B ; \mathbf{Z} / 2 \mathbf{Z}) & \\
\quad & \operatorname{Inv} \operatorname{Lim}\left\{\operatorname{Hom}\left(\operatorname{Hom}\left(H_{q}\left(U_{\alpha}, V_{\alpha} ; \mathbf{Z} / 2 \mathbf{Z}\right), \mathbf{Z} / 2 \mathbf{Z}\right), \mathbf{Z} / 2 \mathbf{Z}\right)\right\}
\end{aligned}
$$

induced by the canonical embeddings of vector spaces into their second duals. This inverse limit is categorically isomorphic to

$$
\operatorname{Hom}\left(\operatorname{Dir} \operatorname{Lim}\left\{\operatorname{Hom}\left(H_{q}\left(U_{\alpha}, V_{\alpha} ; \mathbf{Z} / 2 \mathbf{Z}\right), \mathbf{Z} / 2 \mathbf{Z}\right)\right\}, \mathbf{Z} / 2 \mathbf{Z}\right),
$$

The isomorphism from the Universal Coefficient Theorem [Sp, p. 243]

$$
\operatorname{Hom}\left(H_{q}\left(U_{\alpha}, V_{\alpha} ; \mathbf{Z} / 2 \mathbf{Z}\right), \mathbf{Z} / 2 \mathbf{Z}\right) \cong H^{q}\left(U_{\alpha}, V_{\alpha} ; \mathbf{Z} / 2 \mathbf{Z}\right)
$$

reveals that the last group is

$$
\operatorname{Hom}\left(\check{H}^{q}(A, B ; \mathbf{Z} / 2 \mathbf{Z}), \mathbf{Z} / 2 \mathbf{Z}\right) \text {. }
$$

Since the latter contains an embedded copy of $\check{H}_{q}(A, B ; \mathbf{Z} / 2 \mathbf{Z})$, we conclude that $\check{H}^{q}(A, B ; \mathbf{Z} / 2 \mathbf{Z}) \neq 0$. A final appeal to a Universal Coefficient Theorem [Sp, p. 336] shows that either $\check{H}^{q}(A, B ; \mathbf{Z}) / \neq 0$ or $\check{H}^{q+1}(A, B ; \mathbf{Z}) \neq 0$ and, setting $K=A$, we conclude that $D(K ; \mathbf{Z}) \geq n-$ $k-1$. 


\section{REFERENCES}

[An $\mathrm{An}_{1}$ R. D. Anderson, On monotone interior mappings in the plane, Trans. Amer. Math. Soc., 73 (1952), 211-222.

[An $\left.\mathrm{An}_{2}\right]$, Continuous collections of continuous curves in the plane, Proc. Amer. Math. Soc., 3 (1952), 647-657.

$\left[\mathrm{Dy}_{1}\right]$ E. Dyer, Continuous collections of decomposable continua on a spherical surface, Proc. Amer. Math. Soc., 6 (1955), 351-360.

$\left[\mathrm{Dy}_{2}\right] \quad$, Certain transformations which lower dimension, Ann. of Math., 63 (1956), 15-19.

$\left[\mathrm{Dy}_{3}\right] \quad$, Regular mappings and dimension, Ann. of Math., 67 (1958), 119-149.

[H-W] W. Hurewicz and H. Wallman, Dimension Theory, Princeton University Press, Princeton, N. J., 1941.

[Jo $\left.\mathrm{J}_{1}\right] \quad \mathrm{S}$. Jones, The impossibility of filling $E^{n}$ with arcs, Bull. Amer. Math. Soc., 74 (1968), 155-159.

[ $\left.\mathrm{Jo}_{2}\right] \ldots$, Continuous collections of compact manifolds, Duke Math. J., 37 (1970), 579-587.

[Ku] V. I. Kuz'minov, Homological dimension theory, Russian Math. Surveys, 23 (1968), no. 5, 1-45.

[La] R. C. Lacher, Cell-like mappings and their generalizations, Bull. Amer. Math. Soc., 83 (1977), 495-552.

[L-W] W. Lewis and J. Walsh, A continuous decomposition of the plane into pseudo-arcs, Houston J. Math., 4 (1978), 209-222.

[Na] J. Nagata, Modern Dimension Theory, John Wiley and Sons, Inc. New York, N.Y., 1965.

[Ro] J. H. Roberts, Collections filling a plane, Duke Math. J., 2 (1936), 10-19.

[Sp] E. H. Spanier, Algebraic Topology, McGraw-Hill, New York, 1966.

[Wa $\left.\mathrm{W}_{1}\right]$ J. J. Walsh, Monotone and open mappings on manifolds. I, Trans. Amer. Math. Soc., 209 (1975), 419-432.

$\left[\mathrm{Wa}_{2}\right]$, Light open and open mappings on manifolds. II, Trans. Amer. Math. Soc., 217 (1976), 271-284.

[Wa $\left.\mathrm{Wa}_{3}\right]$, Isotoping mappings to open mappings, Trans. Amer. Math. Soc., 250 (1979), 121-145.

[W-W] J. J. Walsh and D. C. Wilson, The non-existence of continuous decompositions of 3-manifolds into absolute retracts, Houston J. Math., 7 (1981), 591-596.

[Wi $\left.\mathrm{W}_{1}\right] \quad \mathrm{D}$. C. Wilson, Open mappings of the universal curve onto continuous curves, Trans. Amer. Math. Soc., 168 (1972), 487-515.

$\left[\mathrm{Wi}_{2}\right]$ _. Open mappings on manifolds and a counterexample to the Whyburn conjecture, Duke Math. J., 40 (1973), 705-716.

Received February 26, 1982. First author's research partially supported by a National Science Foundation Grant.

UNIVERSITY OF TENNESSEE

KNOXVILLE, TN 37996

AND

UNIVERSITY OF FLORIDA

GAINESVILLE, FL 32611 


\section{PACIFIC JOURNAL OF MATHEMATICS \\ EDITORS}

DONALD BABBITT (Managing Editor)

University of California

Los Angeles, CA 90024

Hugo RossI

University of Utah

Salt Lake City, UT 84112

C. C. MOOre and ARThur OGus

University of California

Berkeley, CA 94720
J. Dugundi

Department of Mathematics

University of Southern California

Los Angeles, CA 90089-1113

R. FINN and H. SAMELSON

Stanford University

Stanford, CA 94305

ASSOCIATE EDITORS

$\begin{array}{lllll}\text { R. Arens } & \text { E. F. Beckenbach } & \text { B. H. Neumann } & \text { F. Wolf } & \text { K. Yoshida }\end{array}$ $(1906-1982)$

\section{SUPPORTING INSTITUTIONS}

UNIVERSITY OF ARIZONA

UNIVERSITY OF BRITISH COLUMBIA

CALIFORNIA INSTITUTE OF TECHNOLOGY

UNIVERSITY OF CALIFORNIA

MONTANA STATE UNIVERSITY

UNIVERSITY OF NEVADA, RENO

NEW MEXICO STATE UNIVERSITY

OREGON STATE UNIVERSITY
UNIVERSITY OF OREGON

UNIVERSITY OF SOUTHERN CALIFORNIA

STANFORD UNIVERSITY

UNIVERSITY OF HAWAII

UNIVERSITY OF TOKYO

UNIVERSITY OF UTAH

WASHINGTON STATE UNIVERSITY

UNIVERSITY OF WASHINGTON 


\section{Pacific Journal of Mathematics}

\section{Vol. 110, No. $1 \quad$ September, 1984}

Wojciech Abramczuk, A class of surjective convolution operators $\ldots \ldots \ldots \ldots 1$

K. Adachi, Extending bounded holomorphic functions from certain

subvarieties of a weakly pseudoconvex domain $\ldots \ldots \ldots \ldots \ldots \ldots$

Malvina Florica Baica, An algorithm in a complex field and its application

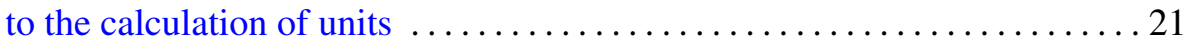

Giuliana Bianchi and Robert Cori, Colorings of hypermaps and a

conjecture of Brenner and Lyndon $\ldots \ldots \ldots \ldots \ldots \ldots \ldots \ldots \ldots \ldots \ldots \ldots$

Ronald James Evans, Determinations of Jacobsthal sums . . .......... 49

Leslie Foged, Characterizations of $\aleph$-spaces .................... 59

Nassif A. Ghoussoub and Paulette Saab, Weak compactness in spaces of

Bochner integrable functions and the Radon-Nikodým property . . . . . . 65

J. Gómez Gil, On local convexity of bounded weak topologies on Banach

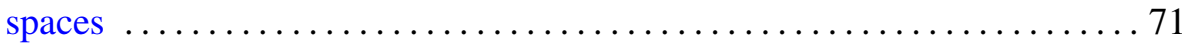

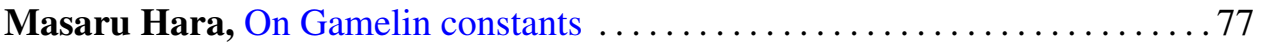

Wilfried Hauenschild, Eberhard Kaniuth and Ajay Kumar, Harmonic

analysis on central hypergroups and induced representations $\ldots \ldots \ldots 83$

Eugenio Hernandez, An interpolation theorem for analytic families of

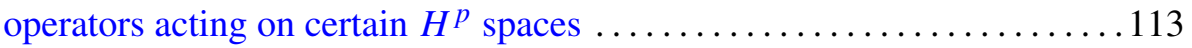

Thomas Alan Keagy, On "Tauberian theorems via block-dominated

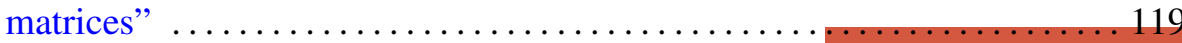

Thomas Landes, Permanence properties of normal structure $\ldots \ldots \ldots \ldots \ldots 125$

Daniel Henry Luecking, Closed ranged restriction operators on weighted

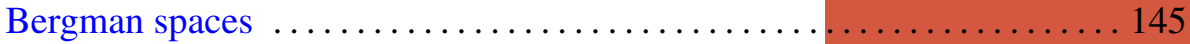

Albert Thomas Lundell, The $p$-equivalence of $\mathrm{SO}(2 n+1)$ and $\mathrm{Sp}(n) \ldots \ldots 161$

Mark D. Meyerson, Remarks on Fenn's "the table theorem" and Zaks' "the chair theorem" ..................................... 167

Marvin Victor Mielke, Homotopically trivial toposes . . . . . . . . . . 171

Gerard J. Murphy, Hyperinvariant subspaces and the topology on Lat A . . 183

Subhashis Nag, On the holomorphy of maps from a complex to a real manifold

Edgar Milan Palmer and Robert William Robinson, Enumeration of self-dual configurations ................................. 203

John J. Walsh and David Clifford Wilson, Continuous decompositions

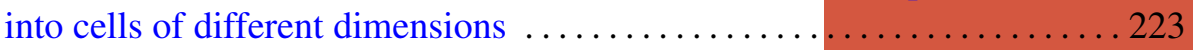

Walter John Whiteley, Infinitesimal motions of a bipartite framework .....233 